\title{
Desarrollo de Competencias Comunicativas de Producción y Comprensión Textual
}

\author{
Development of Communicative Production Competencies and Textual Understanding
}

Lina Marcela Márquez-Galvis ${ }^{1}$

\section{อ} EDICIÓN: :EeEIVTAC

Recibido: 17/julio/2020

Aceptado: 26/agosto/2020

Publicado: 25/septiembre/2020

${ }^{1}$ Colombia

\section{IIIIInstitución \\ ${ }^{1}$ Colegio Agroecológico Holanda}

\section{Correo Eletrónico}

1linamarquez08@gmail.com

\section{ORCID}

'https://orcid.org/0000-0002-0244-4276

\section{Citar así: GCAPA / IEEE}

Márquez-Galvis, L. (2020). Desarrollo de Competencias Comunicativas de Producción y Comprensión Textual. Revista Tecnológica-Educativa Docentes 2.0, $9(2), \quad 158-163$. https://doi.org/10.37843/rted.v9i2.159

L. Márquez-Galvis, "Desarrollo de Competencias Comunicativas de Producción y Comprensión Textual", RTED, vol. 9, n. ${ }^{\circ}$ 2, pp. 158-163, sep. 2020.

\section{Resumen}

La siguiente investigación se realizó bajo la observación, diagnóstico, así como, la planeación y análisis de un proceso que ambiciono fortalecer el desarrollo de competencias comunicativas de producción tal como es la comprensión textual en estudiantes de básica primaria. Inició con un diagnóstico en el nivel de lecto-escritura de los estudiantes, seguidamente, el diseñó de una propuesta de intervención pedagógica a partir de estos resultados; posteriormente se evaluó el efecto de la propuesta de intervención en los procesos de comprensión de textos - producción textual. La investigación se desarrolló bajo el enfoque cualitativo y diseño de investigación acción, dado que son los más acertados en la educación porque los maestros tienen la posibilidad de indagar sobre su propio ejercicio pedagógico; analizarlo y entenderlo, como, el principal instrumento: la autorreflexión. A su vez se involucraron las TIC, con actividades llamativas que no solo permitieran la adquisición de información necesaria, sino también el aprendizaje activo mediante el desarrollo de habilidades como: consulta y manejo de información, desarrollo del nivel crítico, resolución de situaciones y toma de decisiones, creatividad e innovación. A raíz de lo anterior, se diseñaron diversas actividades y estrategias que permitieron fortalecer, ampliación del léxico, trabajo sobre aspectos gramaticales, coherencia, cohesión aplicada a diferentes clases de textos, los cuales contribuyeron al mejoramiento de estos procesos. En conclusión, la intervención implementada mejoró notablemente el desempeño de los estudiantes, tuvo efectos significativos en el rendimiento académico, mejoró el proceso lectoescritor, generó el hábito por la lectura, a su vez permitió una participación dinámica de toda la comunidad educativa.

Palabras clave: Aprendizaje activo, producción y comprensión textual, TIC.

\section{Abstract}

The following research was carried out under the observation, diagnosis, as well as the planning and analysis of a process that aims to strengthen the development of production communication skills such as textual comprehension in elementary school students. It began with a diagnosis of the students' reading-writing level, then the design of a pedagogical intervention proposal based on these results; subsequently, the effect of the intervention proposal on the processes of text comprehension - textual production was evaluated. The research was developed under the qualitative approach and action research design, since they are the most successful in education because teachers have the possibility of inquiring about their own pedagogical exercise; analyze and understand it, as, the main instrument: self-reflection. In turn, ICTs were involved, with striking activities that not only allowed the acquisition of necessary information, but also active learning through the development of skills such as: consultation and information management, development of the critical level, resolution of situations and decision making. decisions, creativity, and innovation. As a result of the above, various activities and strategies were designed that made it possible to strengthen, expand the lexicon, work on grammatical aspects, coherence, cohesion applied to different kinds of texts, which contributed to the improvement of these processes. In conclusion, the intervention implemented notably improved the performance of the students, had significant effects on academic performance, improved the reading-writing process, generated the habit of reading, in turn allowed a dynamic participation of the entire educational community.

Keywords: Active learning, production, text comprehension, ICT. 


\section{Introducción}

El trabajo inició con base en los resultados históricos de las pruebas saber, índice sintético de calidad educativa, análisis del PEI de la institución, aspectos académicos y externos que influyeron significativamente en el nivel en el que se encontraba el Colegio, este es de carácter oficial, ubicado en Colombia-Santander. La I.E presentaba un nivel medio-bajo en cuanto a las pruebas externas Saber, Grado tercero, años -2015-2016 lo que deja ver falencias en los procesos básicos de comprensión y producción textual. (MEN, 2016).

En su momento nació la pregunta: Cuál sería la falencia del alumno- maestro o es posible que los objetivos planteados anualmente son reales y concretos o no se ha podido dar verdadera solución a procesos pedagógicos acordes a las evaluaciones externas. Es de gran importancia resaltar el rol del docente a quienes se "comprometen los recursos, el liderazgo y el saber de los docentes y de las instituciones educativas en un esfuerzo intencional de mejoramiento continuo" (MEN, 2006, p. 6).

La metodología de Investigación-acción surgió de la necesidad de mejorar la comprensión y producción de textos en estudiantes de básica primaria, ya que se observó que los educandos, aunque adquieran conocimientos de diferentes áreas, muestran falencias a la hora de transponer, comprender y producir el tema en otros escenarios o contextos. Dentro de este marco se trabajaron diversas estrategias mediada por las Tecnologías de la Información y la Comunicación (TIC), para lograr el desarrollo de las competencias anteriormente especificadas, teniendo claro que esta se entiende como: Los conocimientos, las habilidades, las actitudes y los valores que se desarrollan en las personas en la interacción con los otros (personas y objetos de conocimiento), son las que les permiten comprender, interactuar y transformar al mundo en el que viven (MEN, 2009).

El trabajo se aplicó con el fin de desarrollar y contribuir al mejoramiento de las competencias de producción textual - oral, como a la competencia de interpretación y comprensión de diferentes clases de textos, para que este proceso conlleve a una calidad educativa. Se realizó mediante la mediación de las TIC, en la cual los estudiantes fueron participantes dinámicos y protagonistas de su propio aprendizaje (activo), bajo estrategias innovadoras; esto con el fin de que de respondieran a las necesidades detectadas y lograran fortalecer los estándares de producción y comprensión textual.

Los efectos que se pretendieron alcanzar es la producción textual oral- escrita, consentido, en la cual se evidencie algunos aspectos gramaticales como (concordancia, tiempos verbales, pronombres) y ortográficos (acentuación, mayúsculas, signos de puntuación) de la lengua castellana (Men, 2006, p. $31)$.

Por consiguiente, no se puede dejar de lado la importancia en el ser integral porque estamos seguras aumentará la autoestima, se establecerán hábitos de estudio, hábitos lectores y el interés por aprender será la mejor estrategia para conseguir la meta planteada de: "lograr estudiantes con una formación permanente, personal, cultural y social" (Ley $\mathrm{N}^{\circ} 115$, 1994). El presente artículo está organizado por Inducción, metodología y resultados.

\section{Metodología}

Este trabajo se realizó siguiendo el enfoque cualitativo y el diseño de investigación acción, con fundamento teórico y conceptual. Esta investigación busca fortalecer el Desarrollo de Competencias Comunicativas de Producción y Comprensión Textual. Es una metodología basada en la importancia del contexto, función y significado de los actos humanos (Martinez, 2000):

Este enfoque estima la importancia de la realidad, tal y como es vivida por el hombre, sus ideas, sentimientos y motivaciones; intenta identificar, analizar, interpretar y comprender la naturaleza profunda de las realidades, su estructura dinámica, aquella que da razón plena de su comportamiento y manifestaciones. Por tanto, lo cualitativo no se opone a lo cuantitativo, sino que lo implica e integra (p. 8).

En este caso parte de la realidad académicacomportamental, en identificar, en analizar, e interpretar, así como comprender para dar soluciones académicas concretas que respondan a los objetivos planteados. Desde otra perspectiva tomamos a Sandin (2000) quien nos habla acerca de criterios en la investigación cualitativa "el entramado es la de interpretación. Los investigadores cualitativos abordan, fundamentalmente, cuestiones relacionadas con acciones, no con actos" (p. 225). 
La clave en el proceso de interpretar todo lo del alrededor, lo cual sucede en el contexto educativo, ya que de estas acciones depende la reflexión y el mejoramiento de la ruta de aprendizaje.

El siguiente apartado contiene conceptualización, características del diseño metodológico investigación acción, así como las orientaciones que se aplicaron para desarrollar la investigación. Se va a desarrollar este apartado con los aportes conceptuales de diferentes autores, las características de la investigación y la explicación de las fases que describirán el proyecto de investigación.

Hablar de investigación acción es iniciar con la definición del concepto y los aportes de diversos autores, Kemmis (1984), Carr y Kemmis (1988), Kemmis y Mctaggart (1992), Elliott (2000), Sandin (2000), Pring (2000), entre otros. Murillo (2010). El término "investigación acción" proviene del autor Kurt Lewin (1994), describía una forma de investigación que podía ligar el enfoque experimental de la ciencia social con programas de acción social que respondiera a los problemas sociales principales de entonces (p. 3). Lo anterior se enfoca sobre las acciones humanas, en este proceso los problemas recobran sentido.

A continuación, se cita a Kemmis (1984) "la investigación-acción no sólo se constituye como ciencia, práctica - moral, sino también como ciencia crítica" (p. 4). Hace énfasis nuevamente en, en la crítica como oportunidad de reflexión de mejora. Espacio para analizar, retroalimentar y definir acciones de cambio y de innovación. "La investigación acción en las escuelas analiza las acciones humanas $y$ las situaciones sociales experimentadas por lo profesores" (Elliott, 1990, p. 24).

Este tipo de investigación da prioridad a la parte humana en toda su expresión, como al rol del docente en su praxis educativa. Elliott (1990) La investigación acción "se relaciona con los problemas prácticos cotidianos experimentados por los profesores en vez de con los "problemas teóricos" definidos por los investigadores puros en torno de una disciplina del saber" (p. 24).

Es de gran importancia entender, que los problemas son los reales, los del diario vivir en aulas, ya que ellos son los que dejan conocer falencias o debilidades tanto cognitivas como influencias sociales. A este respecto hacemos énfasis en las fases de investigación para llevar a cabo en el trabajo de de aula, su correspondiente investigación y análisis, Elliot (1990. p. 32):

1. Primera fase: Identificación de una idea general.

2. Segunda fase: Exploración y planteamiento de la hipótesis.

3. Tercera fase: Construcción del plan de acción.

Además, el método aplicado corresponde a según Elliott (1990) "El método cualitativo, exige un grado de entrenamiento del que normalmente no disponen los profesores" (p. 50). Trabajo esencial en el sistema educativo actual, lo ha dejado de lado, para darle prioridad al cuantitativo, y, así cumplir con las exigencias nacionales e internacionales. Desde otra perspectiva, haciendo énfasis en acción del profesional que aplica este diseño, tomando a Carr (1998) quien afirma:

La competencia profesional requiere capacidad para la deliberación permanente y la discusión critica. Para lograr esa autonomía y responsabilidad, ellos proponen que los propios docentes construyan la teoría de la enseñanza por medio de una reflexión crítica sobre sus propios conocimientos prácticos (p. 2).

Con respecto a lo anterior, la reflexión en el aula es fundamental porque mejora práctica educativa; pero esta acción se produce con una mirada crítica que permita el cuestionamiento y el establecimiento de acciones concretas para mejorar y lograr un excelente proceso de enseñanza- aprendizaje. Asimismo, Pring (2000) señala cuatro características significativas de investigación acción:

Cíclica, recursiva. Pasos similares tienden a
repetirse en una secuencia similar. Participativa.
Los clientes e informantes se implican como
socios, o al menos como participantes activos, en
el proceso de investigación. Cualitativa. Trata más
con el lenguaje que con los números. Reflexiva.
La reflexión crítica sobre el proceso y los
resultados son partes importantes de cada ciclo (p.
7).

Estas características se convierten en el consolidado de la investigación porque se da en espiral, de forma cualitativa en donde el lenguaje, así como la reflexión son su prioridad. Seguidamente se analizó desde otra perspectiva a (Sandin, 2000) el cual afirma: la palabra clave de investigación acción "es la 
de interpretación. Los investigadores cualitativos abordan, fundamentalmente, cuestiones relacionadas con acciones y no con actos, la interpretación del significado de acciones humanas y sociales" (p. 3).

En esta investigación se hace énfasis en interpretación del contexto educativo, partiendo de observación para considerar todos los actos- hechos que generan un significado. Por otra parte, Murillo (2010):

El cual, elabora un modelo para aplicarlo a la enseñanza. El proceso lo organiza sobre dos ejes: uno estratégico, constituido por la acción y la reflexión; y otro organizativo, constituido por la planificación y la observación. Ambas dimensiones están en continua interacción, de manera que se establece una dinámica que contribuye a resolver los problemas y a comprender las prácticas que tienen lugar en la vida cotidiana de la escuela (p. 14).

Seguidamente se trae a colación a Kemmis (1990), para aplicar fases en el proyecto, "está integrado por cuatro fases interrelacionadas: planificación, acción, observación y reflexión. Cada uno de los momentos implica una mirada retrospectiva, una intención prospectiva que forman conjuntamente una espiral autorreflexiva de conocimiento y acción" (p. 14). A propósito, un aspecto fundamental aplicado para dinamizar el proceso, aplicar uso de TIC como espacio para desarrollar competencias tecnológicas, es la sustentada por Mayorga (2020):

\footnotetext{
Las tecnologías del aprendizaje y la comunicación (TAC) orientan las tecnologías de la información y comunicación (TIC) hacia usos formativos; reorientando el proceso de enseñanza -aprendizaje -evaluación hacia el sujeto que aprende (centrado en el estudiante), utilizando un conjunto de servicios, redes, aplicaciones y dispositivos que nos ofrece la era digital dirigidos a mejorar la calidad de los procesos de aprendizaje. (García, W. \&Jaramillo, N. 2020, p. 2.). Seguidamente se habla de la Tecnología "se ha convertido en el motor que mueve el desarrollo del conocimiento desde los orígenes del hombre, el acelerado desarrollo de estas ha generado transformaciones dentro del entorno educativo, jugando un papel primordial en los procesos de enseñanza aprendizaje (p. 1)
}

Así como del aprendizaje activo, el cual los estudiantes son los protagonistas citamos a Mujica (2019) "El Educando tiene un rol mucho activo que, en el modelo tradicional, ya que no solo tiene que escuchar al educador dando alguna explicación, sino que tendrá que regular su propio ritmo de aprendizaje" (p. 1).

\section{Resultados}

Con el desarrollo-ejecución de esta estrategia pedagógica de aula, podemos hacer la siguiente valoración- evaluación. Definitivamente, los docentes tienen una herramienta eficaz para mejorar la comprensión lectora - habilidades para producción oral-escrita. Esta herramienta es implementación de estrategias, donde lo más importante es despertar en el niño el interés por el hacer. Cuando el niño trabaja, estudia, participa con entusiasmo (aprendizaje activo), los resultados fácilmente resaltan a la vista, se puede evidenciar un mejoramiento continuo, cuando estas actividades escolares están basadas en el juego, recreación y tecnología.

El trabajo continuó-reflexivo se dirigió bajo enfoque de "aprendizaje activo, que permitió una mayor interacción y participación de los alumnos" (Sierra y Chocarro 2013, p. 3). En el desarrollo del proyecto se tuvo en cuenta la aplicación de las TIC como instrumento que permitiera realizar lecturas, consultas, actualizaciones, aplicar actividades de Comprensión de lectura en línea, con el fin acercar al estudiante a la Tecnología, pero sobre todo lograr motivación e interés del educando. Como lo expuesto por Mayorga (2020):

\footnotetext{
El uso correcto de las TIC contribuye a la motivación de los educandos, a través de potenciar su creatividad e incrementar la idea de la multitarea, en donde, se aprovecha las sinergias tanto de docentes como de estudiantes, esto fomenta el aprendizaje aumentado en donde, de manera proactiva, autónoma se aprende a sacar partido fortaleciendo las herramientas de internet como fuente de información, recursos, metodologías didácticas-pedagógicas, de gestión e investigación (p. 1).
}

El rendimiento en cada una de las áreas del aprendizaje depende del avance del niño en comprensión de lo leído, depende de estos aspectos desarrolla capacidad para interpretar, comprender y transponer su conocimiento a diversas lecturas o escenarios. Esto unido a una buena producción textual ayudará a mejorar el proceso de enseñanza aprendizaje de los estudiantes. 


\section{Conclusiones}

Al finalizar el proyecto de investigación de producción -comprensión de textos, se concluye que el trabajo se basó en planear -ejecutar estrategias pedagógicas de aula, donde el estudiante interactuó en forma directa con diversos textos, los cuales lo llevaron a fortalecer sus habilidades lecto-escritoras. (Comprensión - producción textual). Durante el proyecto los niños fueron aumentando sus niveles de lectura - escritura, con esta forma fortalecieron su proceso hacia comprender de lo leído. Así mismo se fortalecieron habilidades en producción textual, trazo correcto de palabras-expresión oral, tono de voz, lectura rápida, así como la claridad al leer.

Cada una de estas estrategias permitieron participación directa de los niños (aprendizaje activo), donde el trabajo cooperativo - grupal fue acompañado por actividades lúdicas- recreativas, despertando el interés de los niños lo que hacían más divertidas como entusiastas cada una de jornadas de trabajo lector -escritor. La guía de este trabajo investigativo fueron los lineamientos, pensamientos conceptos de variedad de autores, que dejo ver procesos básicos de comprensión lectora -producción textual, representado en los bajos índices de rendimiento de los niños en diversas pruebas aplicadas por el Ministerio de Educación Nacional (MEN).

En las conclusiones se analizó, que integrar las TIC fue un proceso acertado porque permitió mejorar el problema de los bajos resultados en pruebas externas. En cuanto a las acciones del docente, el maestro debe ser innovador, dinámico, comprometido-orientador del proceso. El docente es el ejemplo de un buen lector y por ende escritor.

En cuanto a las acciones del estudiante se concluye que fue el principal protagonista (aprendizaje activo) en la planificación, el desarrollo de los resultados obtenidos, en cuanto a producción textual, se mejoró el proceso de lecto- escritura básico, aprendieron a realizar escritos siguiendo un plan organizado, teniendo en cuenta el público a quien va dirigido, revisaban, seguidamente corregían sus escritos e incluían nuevo vocabulario.

En relación con la comprensión e interpretación adquirieron la destreza de comprender una lectura. De igual forma desarrollaron competencias tecnológicas, adquiriendo la capacidad de usar de forma pertinente la tecnología como complemento al aprendizaje. Por otro lado, el trabajo sobre las competencias comunicativas de producción y comprensión textual fue muy pertinente porque impactó los procesos cognitivos, procedimentales y actitudinales, aquellos que generan conocimiento, aumenta el léxico, mejora la parte de escritura lo que permite expresar escrito -oralmente sus ideas, pensamientos y sentimientos. Todo esto representado en los resultados finales al escalar un nivel en pruebas externas, de estar en bajo pasó a medio y se espera llegué a alto-superior.

Además, la propuesta constituye una herramienta pedagógica para los docentes que la quieran adoptar, les sirva de apoyo en el mejoramiento de estrategias lectoras para los niños que tengan dificultades en el proceso de lectoescritor. Sintetizando todo lo anterior se concluye que la investigación fue pertinente para contribuir en el mejoramiento del nivel académico-actitudinal $y$ cognitivo de los educandos.

\section{Referencias}

Carr, W. y Kemis, S. (1988). Teoría crítica de la enseñanza. La acción en la formación del profesorado. Barcelona: Martínez Roca. CHACÓN, M.

Elliott, J. (1990). La investigación-acción en educación. Morata.

García, W. \& Jaramillo, N. (2020). Las tecnologías del aprendizaje y la comunicación (TAC) en el marco de la profesionalización docente UNAE-Morona Santiago. Revista Tecnológica-Educativa Docentes 2.0, 9(1), 1216.

Martinez, M. (2000). La investigación Cualitativa etnográfica en educación. Trillas.

Mayorga, M. (2020). Conocimiento, Aplicación e Integración de las TIC -TAC y TEP por los Docentes Universitarios de la Ciudad de Ambato. Revista Tecnológica-Educativa Docentes 2.0, 9(1), 5-1

Mayorga, M. (2020). Conocimiento, aplicación e integración de las TIC - TAC y TEP por los docentes universitarios de la ciudad de Ambato. Revista Tecnológica-Educativa Docentes 2.0, 9(1), 5-11. https://doi.org/10.37843/rted.v9i1.101

MEN. (1994). Ley 115 de febrero 8 de 1994. Recuperado de: http://www.oei.es/quipu/colombia/Ley_115_1994.pdf

MEN. (2006). Resultados ISCE. Bogotá: MEN. Recuperado de: http://www.mineducacion.gov.co/1621/articles345504_anexo_13.pdf 
Desarrollo de Competencias Comunicativas de Producción y Comprensión Textual.

MEN. (2007). Investigación de los saberes pedagógicos. Recuperado de: http://www.mineducacion.gov.co/1621/articles345504_anexo_13.pdf

MEN. (2006). Estándares Básicos de Competencias. Recuperado de: http://www.mineducacion.gov.co/1621/articles-

345504_anexo_13.pdf

MEN. (2006). Estándares Básicos de Competencias. Recuperado de: $\mathrm{http}: / / \mathrm{www}$. mineducacion.gov.co/1621/articles-

345504_anexo_13.pdf

MEN. (2009). El deal educativo del nuevo siglo. Al tableo, pág. 1.

Ministerio de educación. (2016). Colombia Aprende. Recuperado de: http://aprende.colombiaaprende.edu.co/es/agenda/noticias/estr ategias-para-desarrollar-la-comprensi\%C3\%B3n-lectora

Mujica, R. (2019). ¿Cómo organizar una Aula Invertida? Revista Tecnológica-Educativa Docentes 2.0, 5(1), 7-8. Recuperado de: https://ojs.docentes20.com/index.php/revistadocentes20/article/view/67

Murillo, F. (2010). Métodos de Investigación Acción. $3^{\circ}$ edición. Editorial Especias.

Sandin, E. (2000). Criterios de validez en la investigación cualitativa. Revista de la investigación cualitativa, 20.

Sierra, H. (2013). El aprendizaje active como mejora de las actitudes de los estudiantes hacia el aprendizaje. Recuperado de: https://academica-

e.unavarra.es/bitstream/handle/2454/9834/TFM\%20HELENA

$\%$ 20SIERRA.pdf

Torrecilla, F. (2010). Investigación acción. Recuperado de: https://www.uam.es/personal_pdi/stmaria/jmurillo/Investigaci onEE/Presentaciones/Curso_10/Inv_accion_trabajo.pdf 